\title{
Flagellar movement in demembranated preparations of ejaculated fowl spermatozoa
}

\author{
K. Ashizawa, Y. Suzuki and K. Okauchi \\ Laboratory of Animal Reproduction, Faculty of Agriculture, Miyazaki University, \\ Miyazaki 889-21, Japan
}

\begin{abstract}
Summary. Triton X-100 at a concentration of $0 \cdot 1 \%$ in the extraction medium was optimal for demembranating fowl spermatozoa. The most suitable conditions for reactivation were obtained when a medium composed of $0.5 \mathrm{~mm}$-ATP, $25 \mathrm{~mm}$-potassium glutamate, $10^{-7} \mathrm{M}-\mathrm{CaCl}_{2}, 20 \mathrm{~mm}$-Tris- $\mathrm{HCl}(\mathrm{pH} \mathrm{7.9}), 1 \mathrm{~mm}-\mathrm{MgSO}_{4}, 1 \mathrm{~mm}$-dithiothreitol and $0.2 \mathrm{M}$-sucrose was used. More than $60 \%$ motile spermatozoa were obtained under these conditions. The addition of 1 or $10 \mu \mathrm{M}$-cAMP did not appreciably affect motility. Intact and demembranated spermatozoa were immotile at $40^{\circ} \mathrm{C}$, whilst at $30^{\circ} \mathrm{C}$ motility was restored.
\end{abstract}

Keywords: spermatozoa; motility; demembranated models; temperature; fowl

\section{Introduction}

In most synthetic diluents, ejaculated fowl spermatozoa become immotile at a body temperature of 40-41 ${ }^{\circ} \mathrm{C}$ (Munro, 1938; Nevo \& Schindler, 1968; Ashizawa \& Nishiyama, 1978; Takeda, 1982; Wishart \& Ashizawa, 1987). Motility is restored by decreasing the temperature or by the addition of calcium, caffeine or body fluids such as seminal plasma or female peritoneal fluid taken at the time of ovulation (Munro, 1938; Nevo \& Schindler, 1968; Ashizawa et al., 1976; Ashizawa \& Nishiyama, 1978; Takeda, 1982; Ashizawa \& Okauchi, 1984; Ashizawa \& Wishart, 1986, 1987; Wishart \& Ashizawa, 1987). However, the mechanisms of immobilization and restoration of motility have remained unsolved.

To clarify these mechanisms, it may be necessary to establish a demembranated preparation of ejaculated fowl spermatozoa. The advantage of using this preparation is that it permits the study of compounds that do not cross the cell membrane and of agents which have direct effects on the axoneme (White \& Voglmayr, 1986). Furthermore, it may help to clarify whether the plasma membrane is involved in the immobilization of fowl spermatozoa at $40^{\circ} \mathrm{C}$.

Demembranated preparations have been established with the spermatozoa of other species, including sea urchin, fish and many mammals (for reviews, see, for example, Gibbons, 1979; Mann \& Lutwak-Mann, 1981; Tash \& Means, 1983; Morisawa, 1985), but little information is available for avian spermatozoa (Woolley \& Brammall, 1987).

We therefore attempted to determine the most suitable conditions for the demembranation and reactivation of fowl spermatozoa. The effects of temperature on motility of intact and demembranated spermatozoa were also examined.

\section{Materials and Methods}

Animals and preparation of spermatozoa. Commercial White Leghorn roosters (Babcock strain, Akagi Poultry Breeding Farm, Miyazaki, Japan) were used throughout the study. All birds were housed in cages and fed ad libitum on a commercial breeder diet. They were exposed to $14 \mathrm{~h}$ light per $24 \mathrm{~h}$. 
Semen was collected by the method of Bogdonoff \& Shaffner (1954). Samples of semen pooled from 4-6 different males were diluted approximately 10 -fold in $150 \mathrm{~mm}-\mathrm{NaCl}$ with $20 \mathrm{~mm}$-TES ( $N$-Tris-[hydroxymethyl]-methyl-2aminoethanesulphonic acid) at $\mathrm{pH} 7.4$ and centrifuged at $700 \mathrm{~g}$ for $13 \mathrm{~min}$ at room temperature $\left(20-25^{\circ} \mathrm{C}\right)$. The washed spermatozoa were reconstituted in the same buffer to give a final concentration of $\sim 1 \times 10^{9}$ cells $/ \mathrm{ml}$.

Demembranation and reactivation of spermatozoa. The extraction medium and the reactivation medium were based on that used for mammalian spermatozoa (Yeung \& Woolley, 1984), but modified as below; a solution consisting of $0.2 \mathrm{M}$-sucrose, $25 \mathrm{~mm}$-potassium glutamate, $1 \mathrm{mM}-\mathrm{MgSO}_{4}, 1 \mathrm{~mm}$-dithiothreitol (DTT) and $20 \mathrm{~mm}-\mathrm{Tris}-\mathrm{HCl}$ buffer. Various concentrations of Triton X-100 or saponin were added to the extraction medium for demembranation. Various concentrations of ATP, calcium and cAMP were added to the reactivation medium, and the most suitable conditions of demembranation and reactivation for fowl spermatozoa were determined.

To demembranate spermatozoa, $30 \mu \mathrm{l}$ sperm suspension were added to $0.5 \mathrm{ml}$ extraction medium in a test tube. The suspension was incubated for $30 \mathrm{sec}$ while stirring gently with a Pasteur pipette at $20-25^{\circ} \mathrm{C}$ to dissolve the plasma membrane. Then $30 \mu \mathrm{l}$ extraction medium containing demembranated spermatozoa were transferred to $0.5 \mathrm{ml}$ reactivation medium. An aliquant was transferred immediately to a microscope slide chamber $\sim 0.05 \mathrm{~mm}$ deep and a coverslip was placed on the chamber.

The motility of spermatozoa was recorded by videomicroscopy at $20-25^{\circ} \mathrm{C}$ and the percentages of motile spermatozoa were measured by tracing the location of sperm heads on a transparent sheet according to the method of Katz \& Overstreet (1981).

Motility of intact and demembranated spermatozoa at $30^{\circ} \mathrm{C}$ and $40^{\circ} \mathrm{C}$. Intact spermatozoa incubated aerobically for $15 \mathrm{~min}$ at $40^{\circ} \mathrm{C}$ were demembranated at $40^{\circ} \mathrm{C}$. Motility of the demembranated spermatozoa was examined at $40^{\circ} \mathrm{C} \mathrm{on}$ the thermostatically-controlled warm plate according to the method described above. After that, the same samples were cooled to $30^{\circ} \mathrm{C}$ and the motility was observed again. The motility of intact spermatozoa which were suspended in the extraction medium without detergent and transferred to the reactivation medium was also measured.

Electron microscopy. Sperm specimens were suspended in the above extraction medium with Triton $\mathrm{X}-100$ or saponin or extraction medium without detergents for $30 \mathrm{sec}$ and prefixed by adding glutaraldehyde at a final concentration of $2.5 \%$. After $1.5 \mathrm{~h}$, spermatozoa were centrifuged to a pellet and washed in $0.15 \mathrm{M}$-cacodylate buffer without resuspension. The pellet was then post-fixed for $1.5 \mathrm{~h}$ on ice with $2 \%$ osmium tetroxide in the $0 \cdot 1 \mathrm{M}$-cacodylate buffer at $\mathrm{pH} 7 \cdot 4$, dehydrated through a graded series of alcohols and embedded in Spurr's resin. Thin sections were stained first with uranyl acetate and then with lead citrate. Electron micrographs were taken with an Hitachi H-800 electron microscope.

Chemicals. Adenosine 5'-triphosphate (ATP), adenosine 3',5'-cyclic monophosphate (cAMP), phosphodiesterase, potassium glutamate, Triton X-100, dithiothreitol and TES were purchased from Sigma Chemical Co., St Louis, MO, USA. Other chemicals were of reagent grade from Nakarai Chemicals Ltd, Kyoto, Japan.

Statistical analysis. Data were subjected to statistical analysis by the method of Duncan (1955).

\section{Results}

\section{Effects of detergents on the motility of demembranated spermatozoa}

The effects of increasing concentrations of two kinds of detergents, Triton X-100 and saponin, on the motility of demembranated spermatozoa are shown in Fig. 1. Motile spermatozoa were still observed in the extraction medium at concentrations of detergents of $<0.04 \%$. At a higher concentration $(1 \%)$, the percentages of reactivation decreased. Therefore, the concentration of both detergents in the extraction medium was satisfactory at $0.1 \%$ for demembranating the fowl spermatozoa. However, the percentage of reactivation was higher with Triton X-100 treatment than with saponin treatment. Therefore, $0 \cdot 1 \%$ Triton X-100 was used for demembranating the spermatozoa in the later experiments.

After extraction with $0 \cdot 1 \%$ Triton X-100, the plasma membrane was completely removed (Fig. 2). In contrast, saponin-treated spermatozoa still retained the plasma membrane which appeared swollen. This could have been due to a characteristic of saponin which acts selectively on the cholesterol of the plasma membrane, producing small pores in the membrane (Gotoh \& Takenaka, 1984).

\section{Determination of optimal $p H$ and ATP concentration}

The $\mathrm{pH}$ of the Tris- $\mathrm{HCl}$ buffer for extraction and reactivation was varied between $7 \cdot 0$ and $9 \cdot 0$. Reactivation was negligible at $\mathrm{pH} 7 \cdot 0$. The percentages of motile spermatozoa at $\mathrm{pH} 7 \cdot 5-7.9$ 
(a)

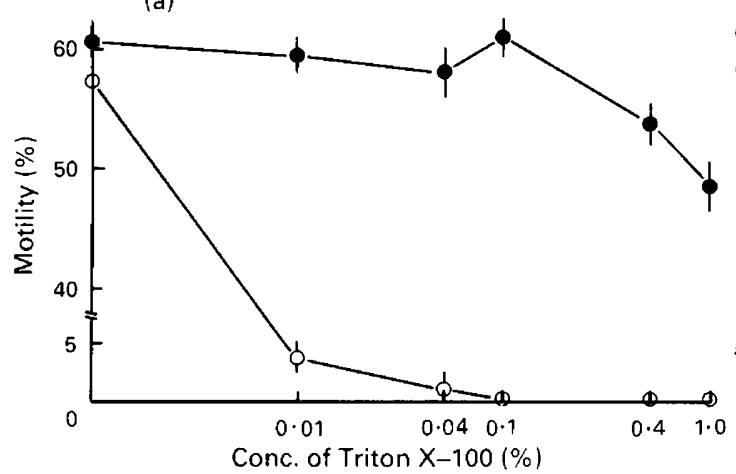

(b)

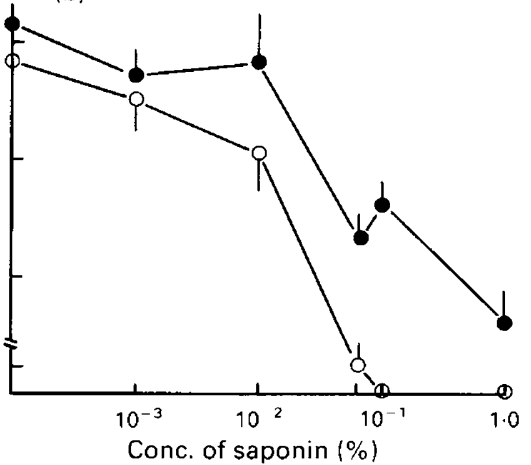

Fig. 1. The motility of the demembranated fowl spermatozoa in the extraction medium $(O)$ or reactivation medium $(\bullet)$. Spermatozoa were demembranated at various concentrations of (a) Triton X-100 or (b) saponin in the extraction medium. ATP concentration and $\mathrm{pH}$ in the reactivation medium were fixed at $0.5 \mathrm{~mm}$ and 7.9 , respectively. Calcium and cAMP were not added. Each point represents the mean ( \pm s.e.m.) motility of 5 samples of spermatozoa.
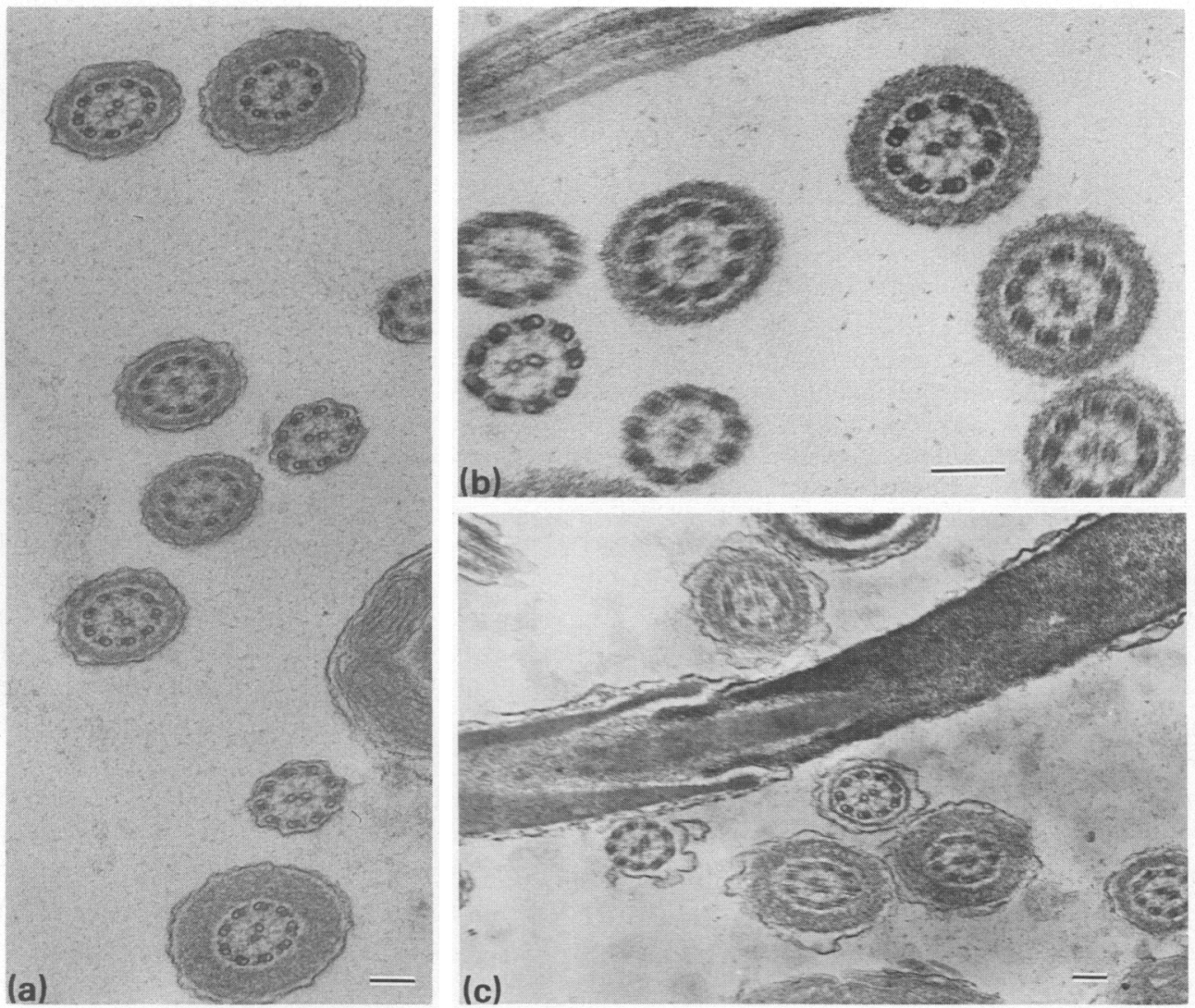

Fig. 2. Electron micrographs of fowl spermatozoa. Spermatozoa were extracted for $30 \mathrm{sec}$ with extraction medium containing (a) no detergent, (b) $0.1 \%$ Triton X-100 and (c) $0.1 \%$ saponin. Bar $=0.1 \mu \mathrm{m}$. 
improved significantly, but those at $\mathrm{pH} 8.5$ and above were lower than that at $\mathrm{pH} 7.9$ (Fig. 3a). The optimal concentration of ATP for reactivation of the spermatozoa was between 0.1 and $0.5 \mathrm{~mm}$. Deviation from this range resulted in a decrease in the percentage of reactivation with no reactivation of spermatozoa from 0 to $0.005 \mathrm{~mm}$-ATP (Fig. 3b).
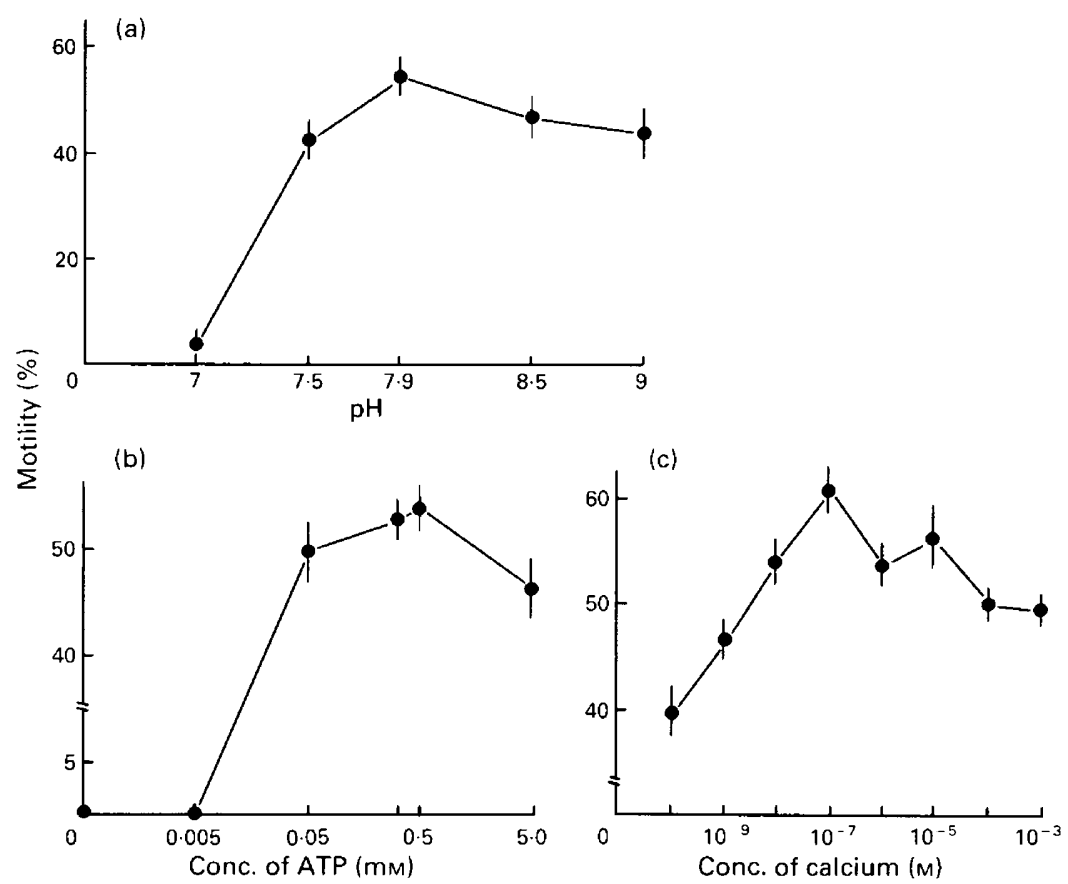

Fig. 3. The effects of (a) $\mathrm{pH}$, (b) ATP concentration and (c) calcium concentration on motility of demembranated fowl spermatozoa in the reactivation medium. In (a) Triton X-100 concentration in the extraction medium and ATP concentration in the reactivation medium were fixed at $0.1 \%$ and $0.5 \mathrm{~mm}$, respectively. Calcium and cAMP were not added. In (b) Triton X-100 in the extraction medium and $\mathrm{pH}$ in the reactivation medium were fixed at $0.1 \%$ and $7 \cdot 9$, respectively. Calcium and cAMP were not added. In (c) calcium concentrations were adjusted by the addition of various concentrations of EGTA. Triton X-100 concentration in the extraction medium, ATP concentration and $\mathrm{pH}$ in the reactivation medium were fixed at $0.1 \%, 0.5 \mathrm{~mm}$ and 7.9 , respectively. Each point represents the mean ( \pm s.e.m.) motility of 5 samples of spermatozoa.

\section{Effects of calcium and cAMP on the motility of demembranated spermatozoa}

As the concentration of calcium was increased, the proportion of motile spermatozoa increased, reaching a peak at $10^{-7} \mathrm{M}$ (Fig. 3c). At concentrations of $>10^{-6} \mathrm{M}$, motility decreased gradually with rise in concentrations, but even at $10^{-3} \mathrm{M}$ about $50 \%$ spermatozoa were still motile.

In the absence of calcium, addition of $1 \mu \mathrm{M}$ - or $10 \mu \mathrm{M}$-cAMP did not stimulate motility (Table 1). In contrast, motility was slightly stimulated in the presence of $10^{-7} \mathrm{M}$-calcium. Addition of phosphodiesterase ( 2 units), to remove endogenous cAMP, gave results similar to those of the control.

The addition of cAMP did not appreciably affect motility of demembranated spermatozoa for at least $15 \mathrm{~min}$ (Fig. 4). 
Table 1. Effects of cAMP on the motility of demembranated fowl spermatozoa in the absence or presence of calcium

\begin{tabular}{lcc}
\hline cAMP conc. $(\mu \mathrm{M})$ & $\mathrm{Ca}^{2+}$ conc. $(\mathrm{M})$ & Motility $(\%)$ \\
\hline 0 & 0 & $50 \cdot 9 \pm 4 \cdot 3^{\mathrm{ab}}$ \\
& $10^{-7}$ & $57 \cdot 7 \pm 2 \cdot 3^{\mathrm{ab}}$ \\
1 & 0 & $52 \cdot 8 \pm 0 \cdot 7^{\mathrm{ab}}$ \\
& $10^{-7}$ & $59 \cdot 1 \pm 3 \cdot 3^{\mathrm{ab}}$ \\
10 & 0 & $49 \cdot 5 \pm 0 \cdot 9^{\mathrm{b}}$ \\
& $10^{-7}$ & $60 \cdot 5 \pm 3 \cdot 1^{\mathrm{a}}$ \\
Phosphodiesterase & $10^{-7}$ & $56 \cdot 8 \pm 3 \cdot 0^{\mathrm{ab}}$ \\
(2 units) & & \\
\end{tabular}

Triton X-100 concentration in the extraction medium, ATP concentration and $\mathrm{pH}$ in the reactivation medium were fixed at $0.1 \%, 0.5 \mathrm{~mm}$ and 7.9 , respectively.

Each value represents the mean ( \pm s.e.m.) of motility of 5 samples of spermatozoa.

Values with different superscripts differ significantly $(P<0.01)$ from each other.

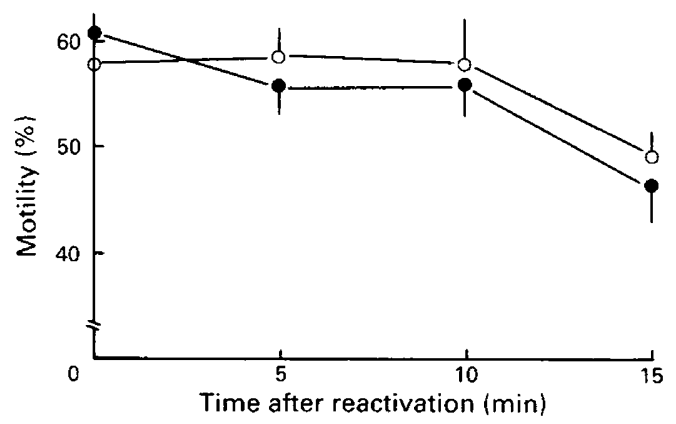

Fig. 4. The effect of cAMP on the maintenance of motility of the demembranated fowl spermatozoa. Triton X-100 concentration in the extraction medium, ATP and calcium concentrations and $\mathrm{pH}$ in the reactivation medium were fixed at $0 \cdot 1 \%, 0.5 \mathrm{mM}, 10^{-7} \mathrm{M}$ and $7 \cdot 9$, respectively. Each point represents the mean $( \pm$ s.e.m.) motility of 5 samples of spermatozoa after the addition of $10 \mu \mathrm{M}-\mathrm{cAMP}(O)$ or no addition $(\Theta)$.

\section{Effects of temperature on the motility of intact and demembranated spermatozoa}

Motility was measured first at $40^{\circ} \mathrm{C}$, and then the same samples were observed at $30^{\circ} \mathrm{C}$. Reactivation of spermatozoa was performed under the most suitable experimental condition, i.e. in a medium composed of $0.5 \mathrm{mM}$-ATP, $25 \mathrm{~mm}$-potassium glutamate, $10^{-7} \mathrm{M}-\mathrm{CaCl}_{2}, 20 \mathrm{~mm}$-Tris- $\mathrm{HCl}$ (pH 7.9), 1 mm- $\mathrm{MgSO}_{4}, 1 \mathrm{~mm}$-DTT and $0 \cdot 2 \mathrm{M}$-sucrose. The motility of intact and demembranated spermatozoa at $40^{\circ} \mathrm{C}$ was raised by reducing the temperature to $30^{\circ} \mathrm{C}$ (Table 2). The addition of cAMP did not restore motility at $40^{\circ} \mathrm{C}$. 
Table 2. Motility of intact and demembranated fowl spermatozoa at $40^{\circ} \mathrm{C}$ and $30^{\circ} \mathrm{C}$

\begin{tabular}{lcc}
\hline \multirow{2}{*}{$\begin{array}{l}\text { Treatment of } \\
\text { spermatozoa }\end{array}$} & \multicolumn{2}{c}{ Motility (\%) } \\
\cline { 2 - 3 } & $40^{\circ} \mathrm{C}$ & $30^{\circ} \mathrm{C}$ \\
\hline Intact spermatozoa & $9 \cdot 9 \pm 2 \cdot 0^{\mathrm{a}}$ & $70 \cdot 0 \pm 4 \cdot 8^{\mathrm{a}}$ \\
Demembranated & & \\
spermatozoa & $2 \cdot 3 \pm 1 \cdot 2^{\mathrm{ab}}$ & $53 \cdot 0 \pm 3 \cdot 9^{\mathrm{a}}$ \\
$\quad+1 \mu \mathrm{M}-\mathrm{cAMP}$ & $1 \cdot 8 \pm 0 \cdot 7^{\mathrm{b}}$ & $53 \cdot 4 \pm 5 \cdot 7^{\mathrm{a}}$ \\
$+10 \mu \mathrm{M}$-cAMP & $2 \cdot 1 \pm 1 \cdot 1^{\mathrm{b}}$ & $53 \cdot 7 \pm 6 \cdot 6^{\mathrm{a}}$ \\
\hline Each value represents the mean $( \pm$ s.e.m.) motility of 5 \\
samples of spermatozoa. \\
Within columns, values with different superscripts differ \\
\multicolumn{2}{l}{ significantly $(P<0 \cdot 01)$ from each other. }
\end{tabular}

\section{Discussion}

These experiments demonstrated that demembranated fowl spermatozoa can be reactivated under suitable conditions. Therefore, it is suggested that demembranated spermatozoa may be useful in clarifying the mechanisms of the reversible temperature-dependent immobilization of fowl spermatozoa. Before we began these experiments, we suspected that the plasma membrane was involved in the immobilization of fowl spermatozoa at body temperature. Therefore, it was assumed that fowl spermatozoa could remain motile at $40^{\circ} \mathrm{C}$ after removing the plasma membrane. However, these results suggest that the mechanisms involved in the immobilization of spermatozoa at $40^{\circ} \mathrm{C}$ do not involve the plasma membrane, because the motility of demembranated spermatozoa was, as with intact spermatozoa, almost negligible at $40^{\circ} \mathrm{C}$ (Table 2). Therefore, it appears that the axoneme itself is directly involved in this immobilization.

The concentrations of detergent and ATP and $\mathrm{pH}$ for optimal reactivation of spermatozoa have been found to vary between species and must be determined in each case. For example, the concentration of Triton X-100 for demembranation of hamster and ram spermatozoa should not exceed $0.05 \%$ in order to preclude the possibility of inhibition of reactivation by ATP (Mohri \& Yanagimachi, 1980; White \& Voglmayr, 1986). Lower concentrations were used for dog (0.01\%) and sea urchin, trout and Cinoma spermatozoa (0.04\%) (Gibbons \& Gibbons, 1972; Tash \& Means, 1982; Morisawa \& Okuno, 1982; Morisawa et al., 1984). In contrast, concentrations of $0.01-0.04 \%$ were insufficient for fowl spermatozoa, because some motile spermatozoa were still observed in the extraction medium. We therefore suggest that a concentration of $0.1 \%$ detergent is optimal for fowl spermatozoa.

The motility of the Triton-treated spermatozoa was completely dependent upon the presence of ATP, and no movement was observed when ATP was omitted from the reactivation medium (Gibbons \& Gibbons, 1972). On the other hand, Yeung (1986) demonstrated that temporary inhibition of the initiation of reactivated motility of hamster spermatozoa was observed by increasing the concentration of ATP from 1 to $3 \mathrm{~mm}$. When the concentration of ATP exceeded the optimum, similar phenomena were observed in dog (Tash \& Means, 1982) and ram (White \& Voglmayr, 1986) spermatozoa. In this study, the optimal concentration of ATP in fowl spermatozoa was obtained between 0.1 and $0.5 \mathrm{~mm}$, and a similar phenomenon was observed at $5 \mathrm{~mm}$.

Regulation of sperm motility by cAMP and calcium is well known (for reviews, see Garbers \& Kopf, 1980; Tash \& Means, 1983; Morisawa, 1985). Demembranated ejaculated bull spermatozoa were most motile in the presence of both ATP and cAMP (Lindemann, 1978). Mohri \& Yanagimachi (1980) also reported that cAMP stimulated the ATP-induced activity of demembranated hamster spermatozoa. Cyclic AMP is also indispensable for the initiation of epididymal sperm motility at 
ejaculation in mammals (Ishida et al., 1987). White \& Voglmayr (1986) routinely added cAMP to the reactivation medium, although the reactivation of ejaculated ram spermatozoa was usually as good with ATP alone. In the present study, cAMP did not affect the maintenance of motility (Fig. 4 ) and the restoration of motility at $40^{\circ} \mathrm{C}$ (Table 2 ). In addition, in the absence of calcium, addition of cAMP alone did not stimulate the motility of ejaculated fowl spermatozoa, although motility was slightly stimulated in the presence of both $10^{-7} \mathrm{M}$-calcium and $10 \mu \mathrm{M}$-cAMP (Table 1).

The effect of calcium on sperm motility is, however, less straightforward. The complexity of the problem probably results from the fact that calcium not only affects cAMP metabolism in spermatozoa, but also has some direct effects on axonemal motility. In addition, external calcium appears to have effects different from those of internal calcium on sperm function, depending on the species (Tash \& Means, 1983). Gibbons \& Gibbons (1980) reported that reactivated sea-urchin spermatozoa show asymmetric beating in the absence of calcium, but in the presence of $100-200 \mu \mathrm{M}$ free calcium the cells became quiescent. The motility of reactivated dog spermatozoa could be stimulated with micromolar levels of cAMP and inhibited with micromolar levels of free calcium (Tash \& Means, 1982). The addition of $10^{-3} \mathrm{M}$-calcium reduced the percentage of reactivation for ejaculated ram spermatozoa (White \& Voglmayr, 1986). In contrast, fowl spermatozoa were still motile even at a concentration of $10^{-3} \mathrm{M}$ free calcium (Fig. $3 \mathrm{c}$ ).

We thank Dr M. Morisawa, Tokyo University and Dr K. Ishida, Teikyo University, Japan, for skilful technical advice; and Mr O. Ravie, AFRC Institute of Animal Physiology \& Genetics Research, Edinburgh Research Station, UK, for kindly reading the manuscript. This study was supported by a grant from the Ministry of Education, Science and Culture, Japan (No. 62760226).

\section{References}

Ashizawa, K. \& Nishiyama, H. (1978) Effects of temperature on the vigour of motility, oxygen consumption and duration of motility of fowl spermatozoa under aerobic conditions. Jpn. Poult. Sci. 15, 264-266.

Ashizawa, K. \& Okauchi, K. (1984) Stimulation of sperm motility and oxygen consumption of fowl spermatozoa by a low molecular weight fraction of seminal plasma. J. Reprod. Fert. 71, 593-598.

Ashizawa, K. \& Wishart, G.J. (1986) Fowl sperm motility stimulating factor(s) from seminal plasma and female peritoneal fluid taken at the time of ovulation. $D e-$ velop. Growth Differ. 28, Suppl. 85, Abstr.

Ashizawa, K. \& Wishart, G.J. (1987) Resolution of the sperm motility-stimulating principle of fowl seminal plasma into $\mathrm{Ca}^{2+}$ and an unidentified low molecular weight factor. J. Reprod. Fert. 81, 495499.

Ashizawa, K., Nishiyama, H. \& Nagae, T. (1976) Effects of oviducal cells on the survival and fertilizing ability of fowl spermatozoa. J. Reprod. Fert. 47, 305-311.

Bogdonoff, P.D., Jr \& Shaffner, C.S. (1954) The effect of $\mathrm{pH}$ on in vitro survival, metabolic activity, and fertilizing capacity of chicken semen. Poult. Sci. 33, 665-669.

Duncan, D.B. (1955) Multiple range and multiple F tests. Biometrics 11, 1-42.

Garbers, D.L. \& Kopf, G.S. (1980) The regulation of spermatozoa by calcium and cyclic nucleotides. $A d v$. cyclic Nucleotide Res. 13, 251-307.

Gibbons, B.H. (1979) Studies on the mechanism of flagellar movement. In The Spermatozoon: Maturation, Motility, Surface Properties and Comparative Aspects, pp. 91-97. Eds D. W. Fawcett \& J. M. Bedford. Urban \& Schwarzenberg, Inc., Baltimore.

Gibbons, B.H. \& Gibbons, I.R. (1972) Flagellar movement and adenosine triphosphatase activity in sea urchin sperm extracted with Triton X-100. J. Cell Biol. 54, 75-97.

Gibbons, B.H. \& Gibbons, I.R. (1980) Calcium-induced quiescence in reactivated sea urchin sperm. J. Cell Biol. 84, 13-27.

Gotoh, H. \& Takenaka, T. (1984) Application of saponized cell model to the study of intracellular organelle motility. Seitai no Kagaku 35, 232-239.

Ishida, K., Okuno, M., Morisawa, S., Mohri, T., Mohri, H., Waku, M. \& Morisawa, M. (1987) Initiation of sperm motility induced by cyclic AMP in hamster and boar. Develop. Growth Differ. 29, 47-56.

Katz, D.F. \& Overstreet, J.W. (1981) Sperm motility assessment by videomicrography. Fert. Steril. 35, 188-193.

Lindemann, C.B. (1978) A cAMP-induced increase in the motility of demembranated bull sperm models. Cell 13, $9-18$.

Mann, T. \& Lutwak-Mann, C. (1981) Biochemistry of spermatozoa: chemical and functional correlations in ejaculated semen, andrological aspects. In Male Reproductive Function and Semen, pp. 195-268. Springer-Verlag, Berlin.

Mohri, H. \& Yanagimachi, R. (1980) Characteristics of motor apparatus in testicular, epididymal and ejaculated spermatozoa. A study using demembranated sperm models. Expl Cell Res. 127, 191-196. 
Morisawa, M. (1985) Initiation mechanism of sperm motility at spawning in teleosts. Zool. Sci. 2, 605-615.

Morisawa, M. \& Okuno, M. (1982) Cyclic AMP induces maturation of trout sperm axoneme to initiate motility. Nature, Lond. 295, 703-704.

Morisawa, M., Morisawa, S. \& De Santis, R. (1984) Initiation of sperm motility in Ciona intestinalis by calcium and cyclic AMP. Zool. Sci. 1, 237-244.

Munro, S.S. (1938) Fowl sperm immobilization by a temperature-media interaction and its biological significance. $Q$. Jl exp. Physiol. 27, 281-287.

Nevo, A.C. \& Schindler, H. (1968) Reversible inactivation of cock spermatozoa by temperature. Proc. 6 th Int. Congr. Anim. Reprod. \& A.I., Paris, Vol. II, 1637- 1640 .

Takeda, A. (1982) Studies on reversible inactivation of cock spermatozoa by temperature. I. Effects of several factors on reversible inactivation. Jpn. Poult. Sci. 19, 26-36.

Tash, J.S. \& Means, A.R. (1982) Regulation of protein phosphorylation and motility of sperm by cyclic adenosine monophosphate and calcium. Biol. Reprod. 26, 745-763.

Tash, J.S. \& Means, A.R. (1983) Cyclic adenosine $3^{\prime}, 5^{\prime}$ monophosphate, calcium and protein phos- phorylation in flagellar motility. Biol. Reprod. $\mathbf{2 8}$ 75-104.

White, I.G. \& Voglmayr, J.K. (1986) ATP-induced reactivation of ram testicular, cauda epididymal, and ejaculated spermatozoa extracted with Triton X-100. Biol. Reprod. 34, 183-193.

Wishart, G.J. \& Ashizawa, K. (1987) Regulation of motility of fowl spermatozoa by calcium and cAMP. $J$. Reprod. Fert. 80, 607-611.

Woolley, D.M. \& Brammall, A. (1987) Direction of sliding and relative sliding velocities within trypsinized sperm axonemes of Gallus domesticus. J. Cell Sci. 88, 361-371.

Yeung, C.H. (1986) Temporary inhibition of the initiation of motility of demembranated hamster sperm by high concentrations of ATP. Int. J. Androl. 9, 359-370.

Yeung, C.H. \& Woolley, D.M. (1984) Three-dimensional bend propagation in hamster sperm models and the direction of roll in free-swimming cells. Cell Motil. 4, 215-226.

Received 30 August 1988 